\title{
Investigation of fluorescence microscopy as a tool for noninvasive detection and imaging of damage precursors at 351-nm
}

S. G. Demos, M. C. Nostrand, M. Staggs, C. W. Carr, D. hahn, M. R. Kozlowski, L. Sheehan, C. Battersby, A. Burnham

Lawrence Livermore National Laboratory

U.S. Department of Energy

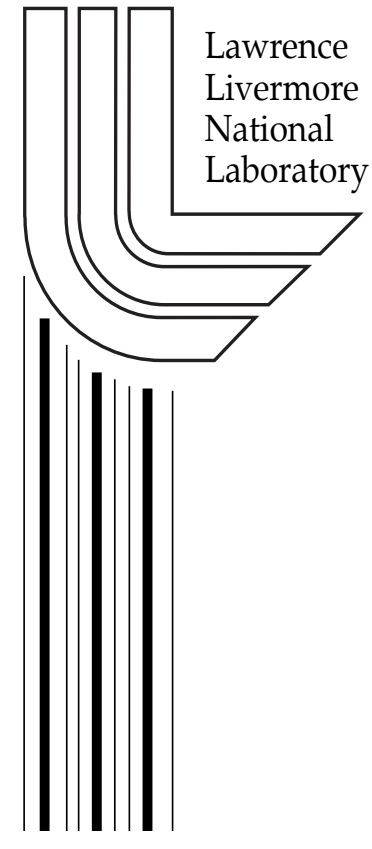

This article was submitted to Laser-Induced Damage in Optical Materials, Nov.1-2, 2001 Boulder, CO.

\section{November 1-2, 2001}




\section{DISCLAIMER}

This document was prepared as an account of work sponsored by an agency of the United States Government. Neither the United States Government nor the University of California nor any of their employees, makes any warranty, express or implied, or assumes any legal liability or responsibility for the accuracy, completeness, or usefulness of any information, apparatus, product, or process disclosed, or represents that its use would not infringe privately owned rights. Reference herein to any specific commercial product, process, or service by trade name, trademark, manufacturer, or otherwise, does not necessarily constitute or imply its endorsement, recommendation, or favoring by the United States Government or the University of California. The views and opinions of authors expressed herein do not necessarily state or reflect those of the United States Government or the University of California, and shall not be used for advertising or product endorsement purposes.

This is a preprint of a paper intended for publication in a journal or proceedings. Since changes may be made before publication, this preprint is made available with the understanding that it will not be cited or reproduced without the permission of the author.

This report has been reproduced directly from the best available copy.

Available to DOE and DOE contractors from the Office of Scientific and Technical Information

P.O. Box 62, Oak Ridge, TN 37831

Prices available from (423) 576-8401 http:/ /apollo.osti.gov/bridge/

Available to the public from the National Technical Information Service

U.S. Department of Commerce 5285 Port Royal Rd., Springfield, VA 22161

http://www.ntis.gov/

\section{OR}

Lawrence Livermore National Laboratory

Technical Information Department's Digital Library

http://www.llnl.gov/tid/Library.html 


\title{
Investigation of fluorescence microscopy as a tool for noninvasive detection and imaging of damage precursors at $351 \mathrm{~nm}$
}

\author{
S. G. Demos ${ }^{*(1)}$, M. C. $\operatorname{Nostrand}^{(1)}$, M. Staggs ${ }^{(1)}$, C. W. $\operatorname{Carr}^{(1)}$, D. Hahn ${ }^{(1)}$, M. R. Kozlowski ${ }^{(2)}$, \\ L. Sheehan ${ }^{(3)}$, C. Battersby ${ }^{(4)}$, A. Burnham ${ }^{(1)}$ \\ ${ }^{(1)}$ Lawrence Livermore National Laboratory, PO Box 808, L-411, Livermore, CA 94551 \\ ${ }^{(2)}$ Currently with Cierra Photonics, 3640 Westwind Blvd., Santa Rosa, CA 95403 \\ ${ }^{(3)}$ Currently with Therma-Wave, 1250 Reliance Way, Fremont, CA 94539 \\ ${ }^{(4)}$ Currently with Terabeam, 12413 Willows Rd. NE, Kirkland, WA 98034
}

\begin{abstract}
This work is an experimental investigation to evaluate the potential of fluorescence microscopy as a tool to detect surface contamination as well as reveal surface damage precursors on DKDP and $\mathrm{SIO}_{2}$ optics. To achieve these technical objectives, microscopic imaging systems were built that also incorporated in-situ damage testing capabilities. Fluorescence imaging experiments were performed using 351-nm laser excitation while damage testing was performed at relatively high laser fluences. The experimental results demonstrated the potential of this technique to address the aforementioned technical issues.
\end{abstract}

Keywords: KDP, DKDP, $\mathrm{SiO}_{2}$, Laser-induced damage

\section{INTRODUCTION}

The detection and characterization of surface defects in various materials in which high purity is critical for their performance has led to a great deal of research and development. This problem was recognized soon after the invention of the laser when laser induced damage initiation at very low fluences was attributed to the presence of mechanical and chemical defects ${ }^{1,2}$. In the fields of high-power laser development and applications, surface quality of the various optical components is of critical importance. Various methods have been explored to remove much of the contamination that led to an increase in the damage threshold ${ }^{3-6}$. However, the damage threshold is still substantially below the intrinsic material limit, especially in large aperture optical components where downselection and/or replacement of optical elements significantly increase the cost of operation ${ }^{7,8}$. To manufacture high damage threshold optics, elimination of damage initiating defect structures located on their surface represents a major challenge. Noninvasive methods to characterize the quality of the surface with respect to its resistance to damage under 355-nm irradiation and pinpoint the location of damage precursors may be the key to address laser induced damage issues.

Fluorescence microscopy utilizes the absorption and emission characteristics of the sample. The most common applications of fluorescence imaging is found in the biomedical field ${ }^{9}$ whereby tissues or cells are "tagged" with particular fluorescing bio-molecules which in turn are used to record images indicating their incorporation into the cells. Most recently, fluorescence microscopy was used to image defect nanostructures located in the bulk of dielectric materials ${ }^{10}$. The presence of emission by a system under laser excitation is associated with the presence of absorption. In wide band-gap optical materials, such as $\mathrm{SiO}_{2}$ and $\mathrm{KDP}$, there should be no absorption, and therefore emission, at $355-\mathrm{nm}$. However, the observed laser induced damage at relatively low fluence may be suggestive of the presence of absorbing defects such as foreign particles, etc. This indicates that fluorescence microscopy may be a suitable tool for the detection of absorbing defect structures in optical materials that may be the cause of laserinduced damage.

The objective of this work was to investigate fluorescence microscopy as a tool that could provide information regarding the quality of optical surfaces by detecting the presence of foreign substances. The observed defect structures could then be exposed to high power laser irradiation to reveal their potential to initiate damage. In this report, we will describe our preliminary effort that involved fluorescence imaging of $\mathrm{SiO}_{2}$ and $\mathrm{KDP}$ surfaces and in situ damage testing experiments.

*Correspondence: Email: Demos1@1lnl.gov; Telephone: 925423 3388; Fax: 9254232463 


\section{EXPERIMENTAL SET-UP}

A detailed description of the experimental apparatus utilized in this work can be found elsewhere ${ }^{11}$. The experiments were performed on polished fused silica and diamond-turned, conventionally and rapidly grown DKDP crystals. All sample surfaces were flat. The experiments were performed with the samples held at room temperature.

\section{FLUORESCENCE IMAGING ON $\mathrm{SiO}_{2}$ SURFACES}

Fluorescence images of $\mathrm{SiO}_{2}$ surfaces were recorded and compared. The various samples tested had undergone different polishing processes culminating in a wide range of surface damage thresholds the results of which are listed in Table 1 along with the information regarding the polishing process and the corresponding descriptive damage threshold. In addition, Figure 1 is the Ramped-on-1 (R/1) probability damage plot versus fluence for each sample listed in Table 1. Thus, Figure 1 shows how the descriptive average damage thresholds for Table 1 were formulated (i.e., very low for sample L-120-1 to high for sample 6K03).

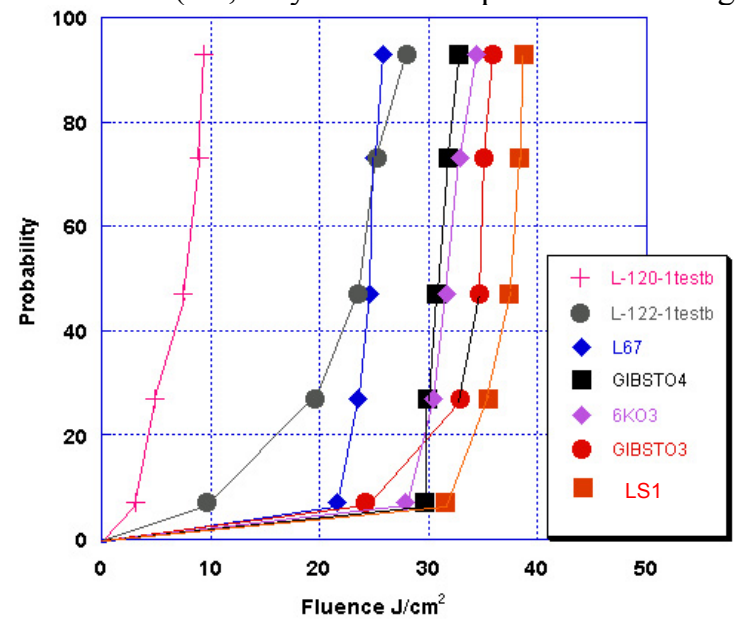

Figure 1: R/1 probability damage plot versus fluence for each sample listed in Table 1.

\begin{tabular}{|c|c|c|c|}
\hline Part \# & Polish & $\begin{array}{c}\text { Subsurface } \\
\text { damage }\end{array}$ & Average DT \\
\hline L-120-1 & $\mathrm{Ce}$ & High & Very low \\
\hline L-122-1 & $\mathrm{Ce}$ & High & Low \\
\hline L67 & $\mathrm{Ce}$ & Med & Low \\
\hline GIBST04 & $\mathrm{Ce}+$ & None & High \\
\hline GIBST03 & $\mathrm{Zr}$ & None & High \\
\hline LS1 3/97 & unknown & None & High \\
\hline $6 \mathrm{~K} 03$ & $\mathrm{Ce}+\mathrm{Ion}$ Mill & None & High \\
\hline
\end{tabular}

Table 1: $\mathrm{SiO}_{2}$ samples utilized to obtain fluorescence images of their surface.

Figure 2 shows microscopic fluorescence images (MFI) of the surface of the samples (10x objective with a $5 \mathrm{x}$ zoom). The contrast on these images has been adjusted to better show the existing variation on the emission intensity from the different points of the surface. The average emission intensity from the surface of each sample was different between samples and it was found to depend on the polishing process. The Ce polished samples exhibited maximum emission that appeared to scale with the damage threshold of the material. The highest damage threshold samples exhibit very low emission with relatively uniform distribution while features on the surface that exhibit significantly higher intensity are rare. Dark scratches and points are observed on sample 6K03. Few features with some dark scratches are observed on sample GIBST03. Sample LS13/97 shows also a clean surface exhibiting low emission. Sample GIBST04 shows the presence of emissive lines and points with somewhat higher intensity. The above samples have relatively high damage thresholds. On the other hand, low damage threshold samples exhibit a different behavior. Sample L122-1 exhibits a speckled background and bright points having diameters of the order of a few microns. Sample L67 shows the presence of a high concentration of bright points. Finally, the image of the surface of sample L120 indicates the presence of a web of cracks that are filled with an emissive material. The images of the surfaces in Figure 2 are arranged according to their measured damage threshold (high to low, left to right, top to bottom). An examination of the surface morphology as depicted by the fluorescence images suggests a reasonable correlation of the presence of emissive features on the surface with a lowering of the damage threshold.

Comparison of the fluorescence images with the light scattering images of the same section of the samples indicate that the observed features using fluorescence microscopy are not always visible in the corresponding light scattering image. This is demonstrated in Figures 3 and 4. Figure 3 indicates that the fluorescence image contains a much smaller number of features than the corresponding light scattering image. Some of these features can be observed using light scattering. Two lines observed in the fluorescence image are presumably cracks or scratches filled with an emissive material. These features are not visible using light scattering. 

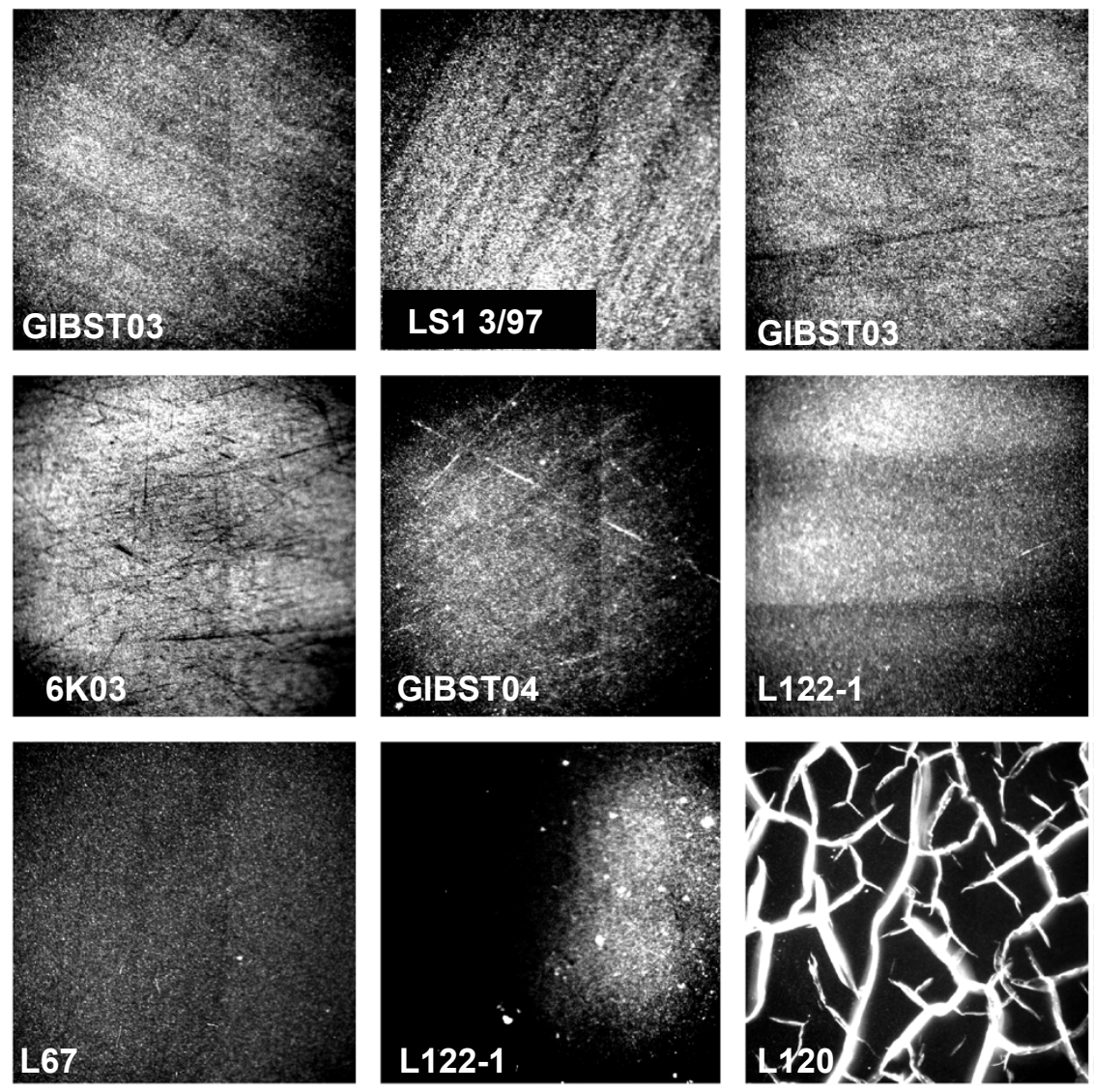

Figure 2: Microscopic fluorescence images of the surface of $\mathrm{SiO}_{2}$ samples under $351 \mathrm{~nm}$ excitation. Each image measures $1.2 \mathrm{~mm}$ per side.
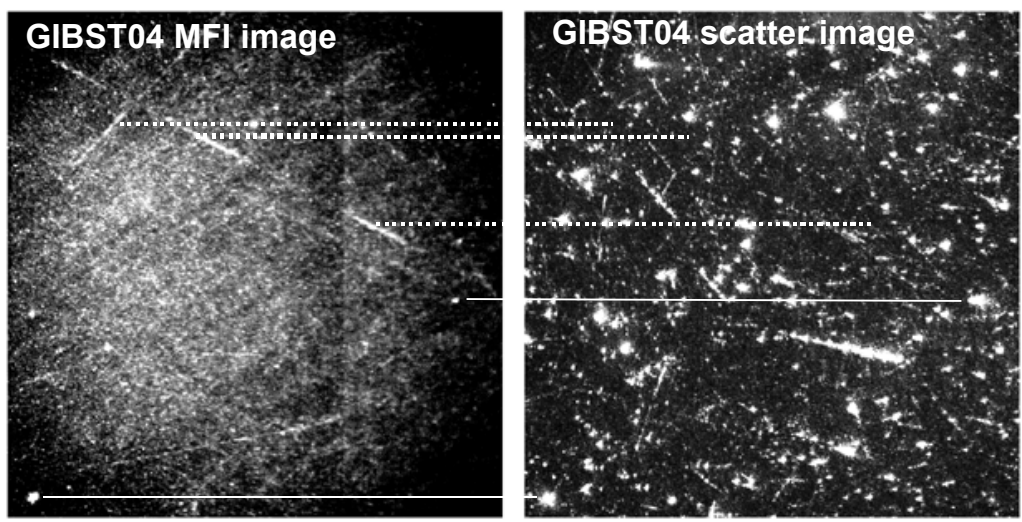

Figure 3: Fluorescence and light scattering images of the same section of the surface of $\mathrm{SiO}_{2}$ sample GIBST04. Each image measures $1.2 \mathrm{~mm}$ per side.
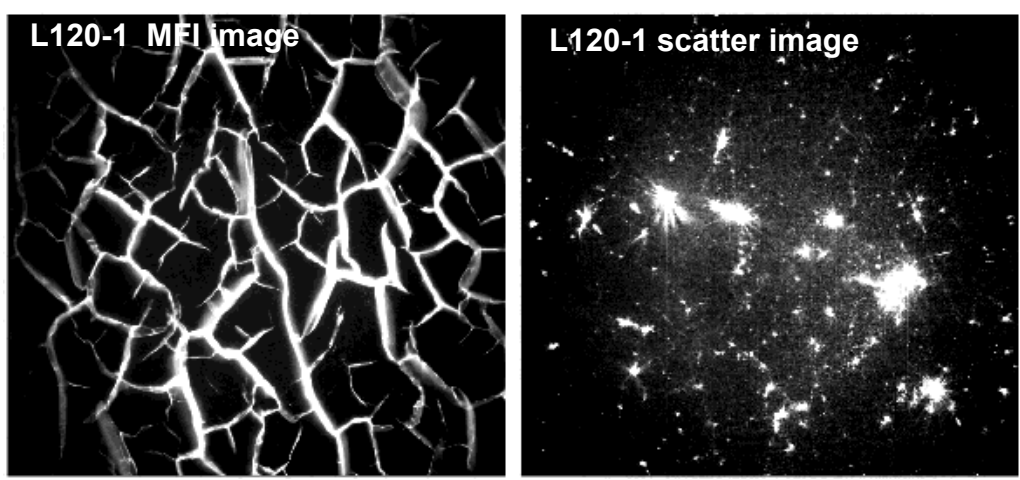

Figure 4: Fluorescence and light scattering images of the same section of the surface of $\mathrm{SiO}_{2}$ sample L120-1. Each image measures $1.2 \mathrm{~mm}$ per side. 
Similarly, Figure 4 shows that the web of fluorescing cracks in sample L-120-1 is totally invisible in the corresponding light scattering image.

An obvious question arising from these experimental results is if the observed fluorescing features are responsible for damage initiation. To address this issue, experiments were performed using a Spectra-Physics commercial laser operating at $355-\mathrm{nm}, 7.5-\mathrm{ns}$, with fluences up to $\sim 40 \mathrm{~J} / \mathrm{cm}^{2}$. Figure 5 shows a typical example of the experimental data. The fluorescence image of the sample prior and following irradiation with the 355 -nm laser pulses is shown. Damage sites are clearly visible due to the emission from the modified material. An examination of the location where damage was initiated with respect to the location of preexisting fluorescing features indicates reasonable correlation. There are damage sites that were initiated at the exact location where fluorescing features were observed prior to irradiation. However, there are a number of damage sites that were initiated at locations where there were no dominant features in the fluorescence image. It must be pointed out that all experiments on $\mathrm{SiO}_{2}$ samples were performed at the early stages of this effort when instrumentation and methodology were still not optimized. It is therefore difficult to conclude if the initiators of the damage sites that do not correlate with the fluorescence map is not visible using fluorescence microscopy or if their sizes are so small that their emission signals are below the detection limit of the system. In any case, the correlation of damage initiation (at 7.5-ns, 40 $\mathrm{J} / \mathrm{cm}^{2}$ ) with preexisting features was much better for the fluorescence microscopy approach than for light scattering at the time that the experiments were performed.
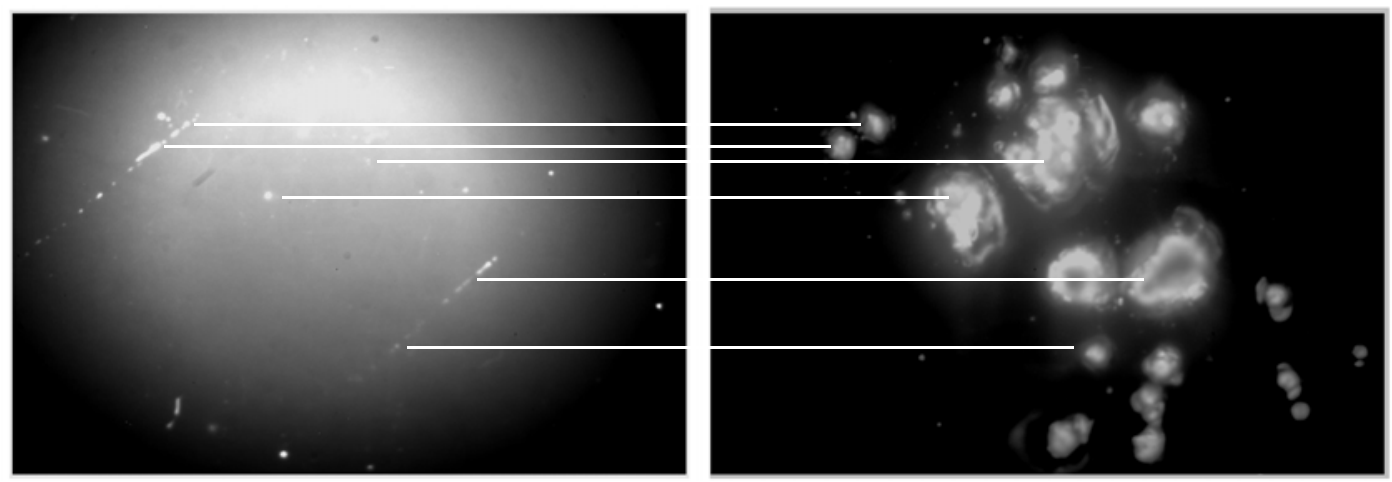

Figure 5: Fluorescence images of the surface of the $\mathrm{SiO}_{2}$ sample prior and following irradiation with a 355-nm, 7.5 ns laser pulse at $\approx 40 \mathrm{~J} / \mathrm{cm}^{2}$. The horizontal dimension in these images is $1.2 \mathrm{~mm}$.

\section{FLUORESCENCE IMAGING OF DKDP SURFACES}

Two deuterated KDP (DKDP) samples with characteristically different surface damage behavior were studied using fluorescence imaging. These were samples RG8A, a third-harmonic-generation crystal with greater than $80 \%$ deuteration, and LL3-LG, a crystal cut normal to the extraordinary axis with $70 \%$ deuteration, which are rapid and conventional growth, respectively. The $\mathrm{R} / 1$ probability damage plot versus fluence for the above samples are shown in Figure 6. Figure 7 shows typical fluorescence and light scattering images from DKDP sample RG8A. This sample has a very low surface damage threshold. The main features in the fluorescence image are not present in the light scattering image. This indicates that these fluorescing objects are located below the top layer of the surface. Using maximum optical magnification (X100 microscope objective) the estimated dimension of these features was $\approx 100 \mu \mathrm{m}$ in length, $\approx 8 \mu \mathrm{m}$ in width and, $\approx 50 \mu \mathrm{m}$ in depth.

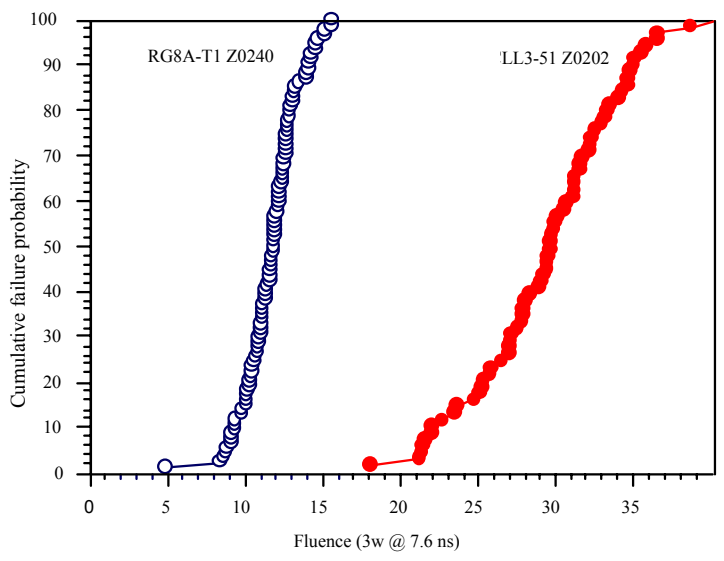

Figure 6: R/1 probability damage plot versus fluence for DKDP samples RG8A and LL3-LG 
Sample LL3-LG has a high surface damage threshold. Typical fluorescence and light scattering images from this sample are shown in Figure 8. Fluorescing spots are observed on the surface of this sample that do not correlate with the observed features in the light scattering image. Comparison of the light scattering images from the two samples indicates that sample LL3-LG has a much cleaner surface. In addition, the fluorescing objects in sample RG8A are much bigger and brighter compared to those observed in sample LL3-LG. The background emission observed in the fluorescence images is due to the nearly uniform emission from the surface of the samples while the structure observed is due to the nonuniformity of the 351-nm illumination beam. Examination of the surface of these samples indicated that it is rather common to observe features that are visible to both imaging approaches. This is attributed to the presence of contamination particles that are on the surface of the samples that are also emissive under 351-nm laser photoexcitation. The presence of emissive structures embedded into the surface of the material (not visible with light scattering) suggests the incorporation of contaminants during the polishing process and/or subsequent handling. These results suggest that fluorescence microscopy may provide a way of monitoring the degree of contamination. Furthermore, the observation of large size, highly emissive features on the surface of sample RG8A is consistent with its low surface damage threshold.
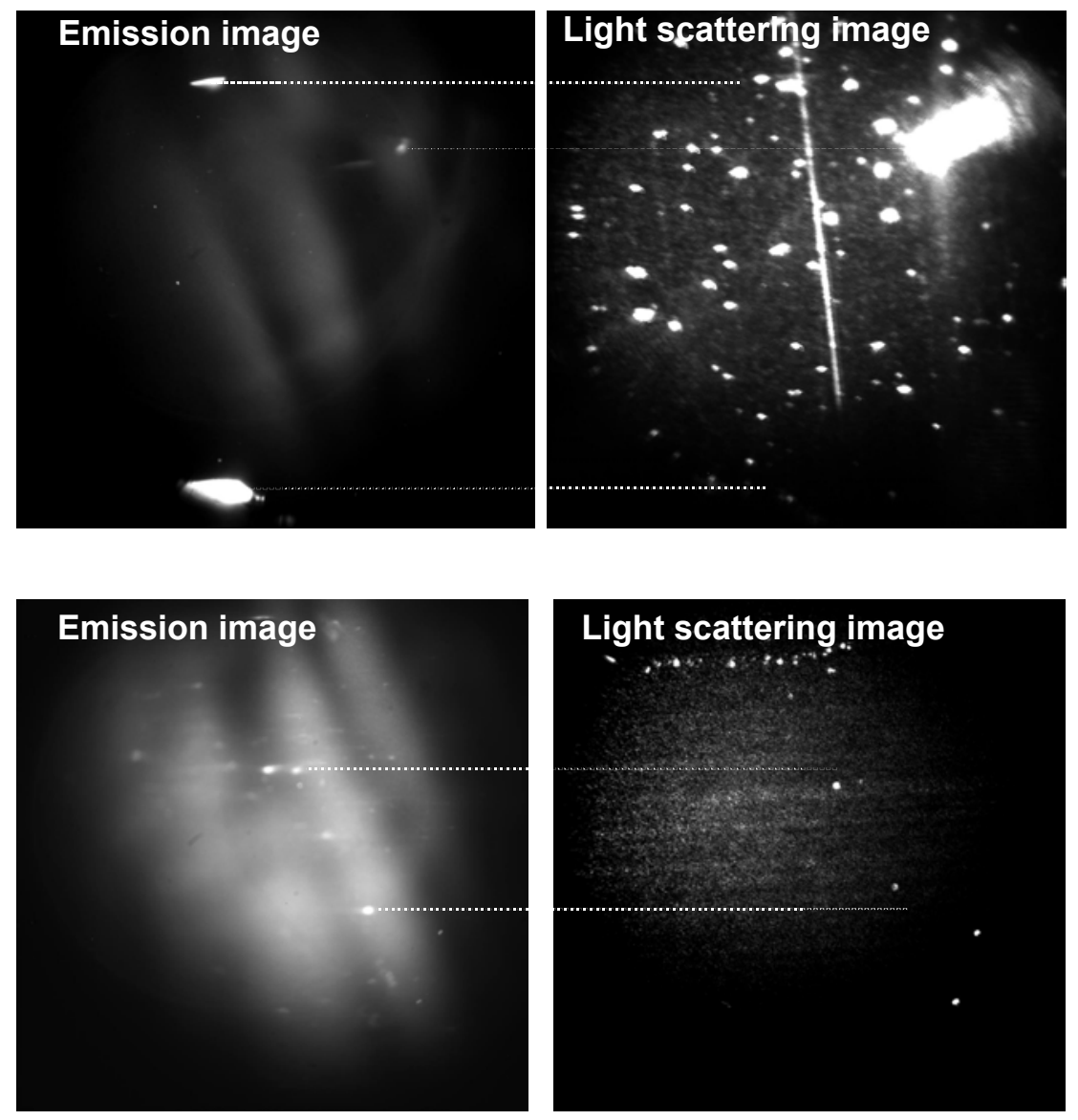

Figure 7: Fluorescence and light scattering images of the same $1025 \mathrm{X} 1025 \mu \mathrm{m}^{2}$ section of the surface of DKDP sample RG8A

Figure 8: Images of the same $1025 \times 1025 \mu^{2}$ section of the surface of DKDP sample LL3-LG

\section{IN-SITU DAMAGE TESTING OF DKDP SURFACES}

In-situ damage testing experiments were performed on samples RG8A and LL3-LG. The objective was to irradiate objects observed using fluorescence microscopy with 355-nm laser pulses and examine their susceptibility to laser induced damage initiation.

Figure 9-1 shows a light scattering image from the surface of sample RG8A before irradiation. The corresponding fluorescence image under $351-\mathrm{nm}, \mathrm{CW}$ excitation is shown in Figure 9-2. These images were obtained before the sample was irradiated with the high fluence, 355-nm laser pulse. Figure 9-3 shows the captured image during irradiation with a single $3-\mathrm{ns}, 355-\mathrm{nm}$ pulse at $15 \pm 3 \mathrm{~J} / \mathrm{cm}^{2}$. The light scattering image after irradiation with the 355-nm pulse shown in Figure 9-4 demonstrates the formation of a damage site as a result of the exposure 
of the sample to the laser pulse. The location of the damage site is identical to the location where light was observed during irradiation (see Figure 9-3) and the location of the preexisting large fluorescing object (see Figure 9-2). This experiment showed the correlation of the preexisting feature visible only using fluorescence microscopy with damage initiation. The detected light during irradiation (Figure 9-3) is presumably due to plasma formation during damage. The same experiment was repeated using the same sample and four additional fluorescing features, similar to those shown in Figures 9-2 and 8, were examined. In total, 5 sites were chosen and in all cases, damage was initiated within the area where the observed features were located. All experiments were performed using $\approx 15 \mathrm{~J} / \mathrm{cm}^{2}$ laser fluence at $3 \mathrm{~ns}$.
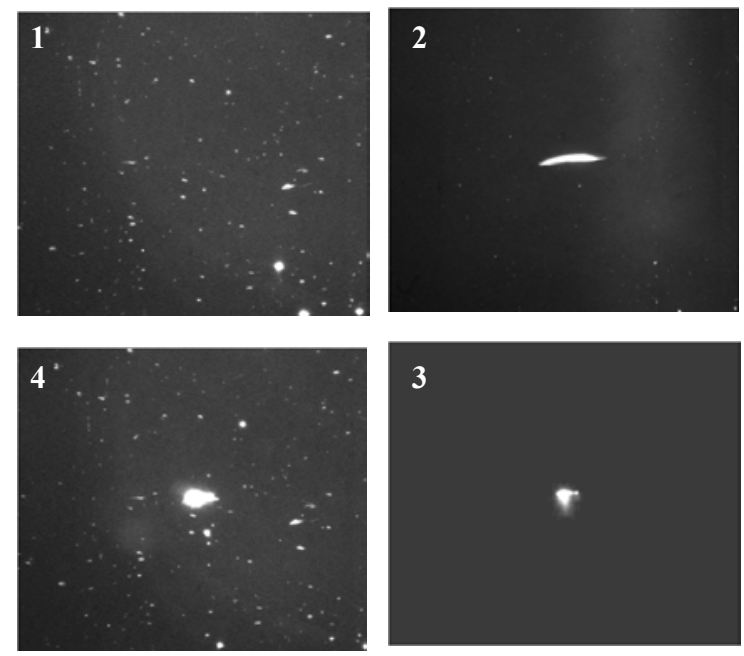

Figure 9: Images from same $600 \times 520 \mu \mathrm{m}^{2}$ section of the surface of DKDP sample RG8A
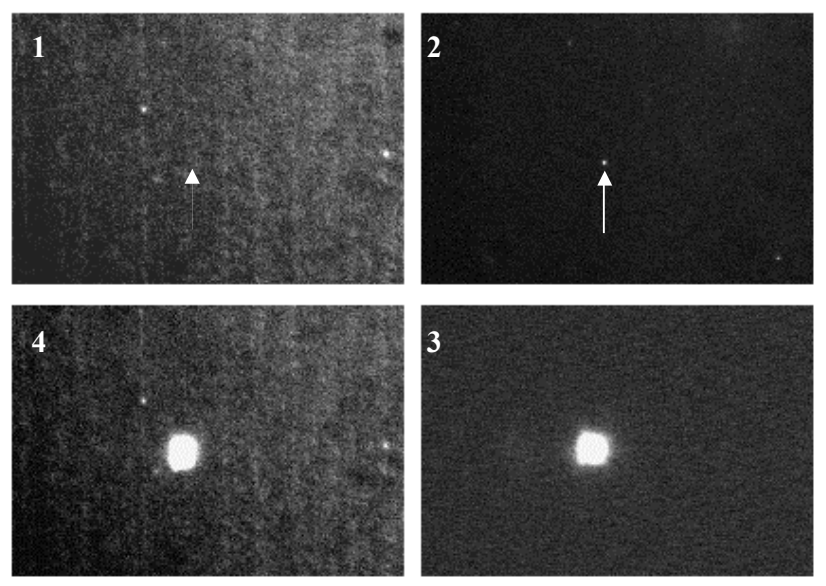

Figure 10: Images from same $420 X 280 \mu \mathrm{m}^{2}$ section of the surface of DKDP sample LL3-LG.

1) Light scattering image before irradiation. 2) Fluorescence image under 351-nm, excitation. 3) Plasma emission image during irradiation with a single 3 -ns, $355-\mathrm{nm}$ pulse at $\approx 15 \mathrm{~J} / \mathrm{cm}^{2} .4$ ) Light scattering image after irradiation with a single $355-\mathrm{nm}$ pulse.

Similar experiments were performed on sample LL3-LG. Fluorescence features were irradiated with $\approx 15 \mathrm{~J} / \mathrm{cm}^{2}$, 3-ns, 355-nm pulses while the behavior was monitored during this process. More than 40 sites over a two-week period were targeted and tested at $\approx 15 \mathrm{~J} / \mathrm{cm}^{2}$. In all but one case, fluorescence features did not correlate with an observable reaction due to the $355-\mathrm{nm}$ laser irradiation that can be classified as laser induced damage. In most of the cases, the intensity of the fluorescence feature was observed to be reduced after laser irradiation. The only location resulting in damage that was clearly visible using light scattering is shown in Figure 10. Typical reactions of other fluorescing features at other locations to laser irradiation will be shown in later figures.

Figures 10-1 and 10-2 show a light scattering image and the corresponding fluorescence image, respectively, from the surface of sample LL3-LG before irradiation. Note that the fluorescence object is not visible in the light scattering image. Figure 10-3 shows the plasma image recorded during irradiation with a $355-\mathrm{nm}$ pulse at $\approx 15 \mathrm{~J} / \mathrm{cm}^{2}$. The light scattering image after irradiation with the $355-\mathrm{nm}$ pulse (Figure 10-4) indicates the presence of damage and a correlation with the pre-existing fluorescence "object". Thus, the only damage initiation we were able to record during the course of this experiment on sample LL3-LG is suggesting that the fluorescing defect was the damage initiator.

The presence of emission is evidence for absorption. It has been suggested that an absorbing particle can heat up to thousands of degrees and cause damage initiation. ${ }^{12}$ In this case, it is obvious that the absorbing particle needs to be appropriately coupled in to the host material so that its excess energy is efficiently transferred in the form of heat or a pressure wave into the host. It was one objective of this preliminary work to prove that fluorescing objects can respond in a nonreversible and dynamic manner to exposure to high fluence laser irradiation without leading to damage of the host material. Experimental evidence of this effect was recorded during our study of sample LL3-LG. 
Figure 11-1 shows the light scattering image of the surface of the sample with an object of interest indicated with an arrow. Figure 11-2 shows the fluorescence image of the same area where the object of interest is clearly visible. During irradiation with a single laser pulse, formation of plasma was recorded as shown in Figure 11-3. The light scattering image after irradiation (Figure 11-4) suggests no damage to the host material as a result of plasma formation. The only clear difference is that the size of the object has been reduced. However, the fluorescence image shown in Figure 11-5 is noticeably different from that shown in Figure 11-2. A contaminated fluorescing area around the particle that led to plasma formation is observed. This is indicative of a micro-explosion that was associated with the generation of mobile ionic and/or molecular species that were spattered into the surrounding area. Subsequent 355-nm irradiation led to no additional formation of plasma or change in the fluorescence images. This is demonstrated in Figure 11-6 where the fluorescence image following irradiation with 10 pulses is shown. A long arrow shaped feature fluorescing adjacent to the object of interest did not initiate damage. The only noticeable change of this object as a result to laser irradiation was the reduction of its intensity as demonstrated in figs. 11-2, and 11-5.
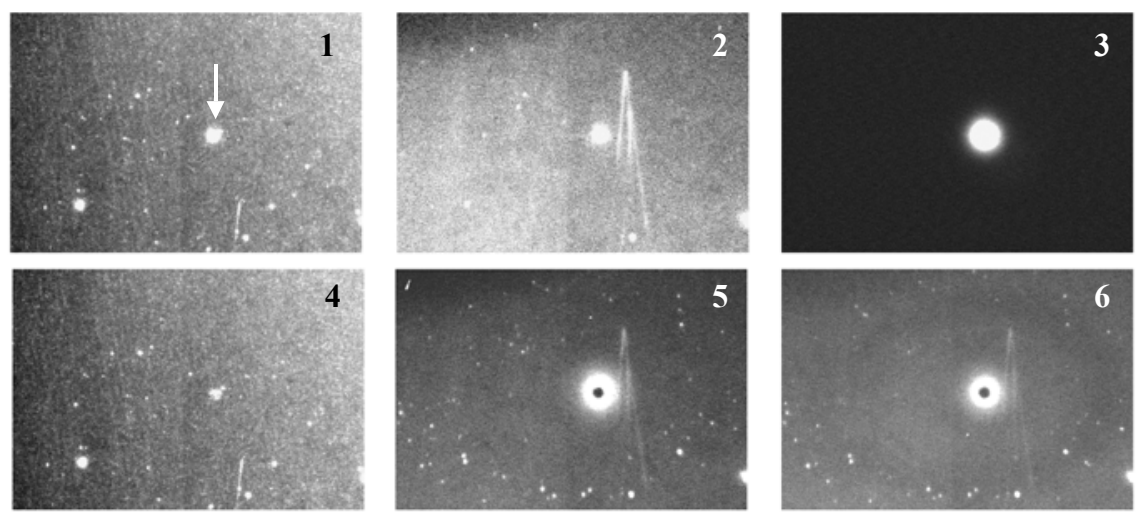

Figure 11: Images from same $450 \times 310 \mu \mathrm{m}^{2}$ section of the surface of sample LL3-LG showing the reaction of surface defects from contamination to $355 \mathrm{~nm}, 3 \mathrm{~ns}$ laser pulses at $\approx 15 \mathrm{~J} / \mathrm{cm}^{2}$. Details are provided in the text
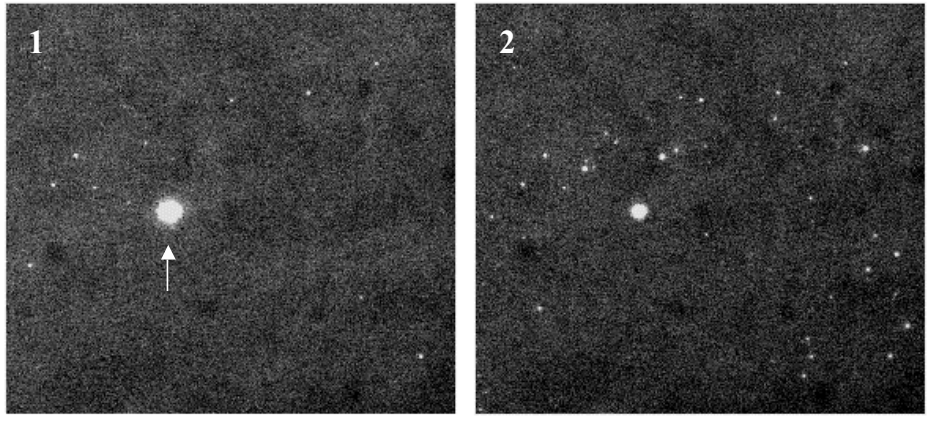

Figure 12: Light scattering images from same $290 \mathrm{X} 260 \mu \mathrm{m}^{2}$ section of the surface of sample LL3-LG (1) before and (2) after irradiation with a single laser pulse at $\approx 15$ $\mathrm{J} / \mathrm{cm}^{2}$.

A similar effect is demonstrated in Figure 12. The object of interest is denoted with an arrow in the fluorescence image (Figure 12-1) acquired before irradiation of the sample. This object was very bright with a maximum pixel intensity of $\approx 6000$ counts and it was not visible in the light scattering image. Figure 12-2 shows the fluorescence image after irradiation. The intensity of the object has now been reduced to $\approx 200$ counts/pixel while a number of new particles are observed on the image. This suggests the original particle "exploded" creating a number of smaller ones. Post irradiation examination of the sample with light scattering does not indicate the presence of damage. 



\section{FLUORESCENCE IMAGING AND DAMAGE TESTING OF A 15-cm DKDP CRYSTAL}

Recently, higher quality $15-\mathrm{cm}$ DKDP ( $80 \%$ deuteration, to be used for 351-nm harmonic generation) became available for experimentation. These materials were considered to be from the best production and handling methods available at the time. Such material is desirable to avoid unnecessary contamination and/or irrelevant damage precursors. One crystal of this set, taken from rapid-growth production boule BD8-10 and finished with a single-point diamond turning machine, is the subject of this section. The fluorescence imaging technique was used on this sample in an attempt to identify and correlate surface damage precursors.

The experimental plan consisted of constructing a fluorescence image map of the sample before and after exposure to 351-nm laser pulses, then correlate the surface damage features in the 'after' map to the fluorescent features in the 'before' map. The laser beam was that of the Optical Sciences Laser (OSL), consisting of 3-cm diameter, 3-ns flattop pulses. A total of eight OSL footprints were applied to the sample. In order to test the environmental effects of surface damage, four of these footprints were associated with an environment of 760 torr (air), while the other four were at 1 torr. Figure 13 shows the laser footprint configuration.

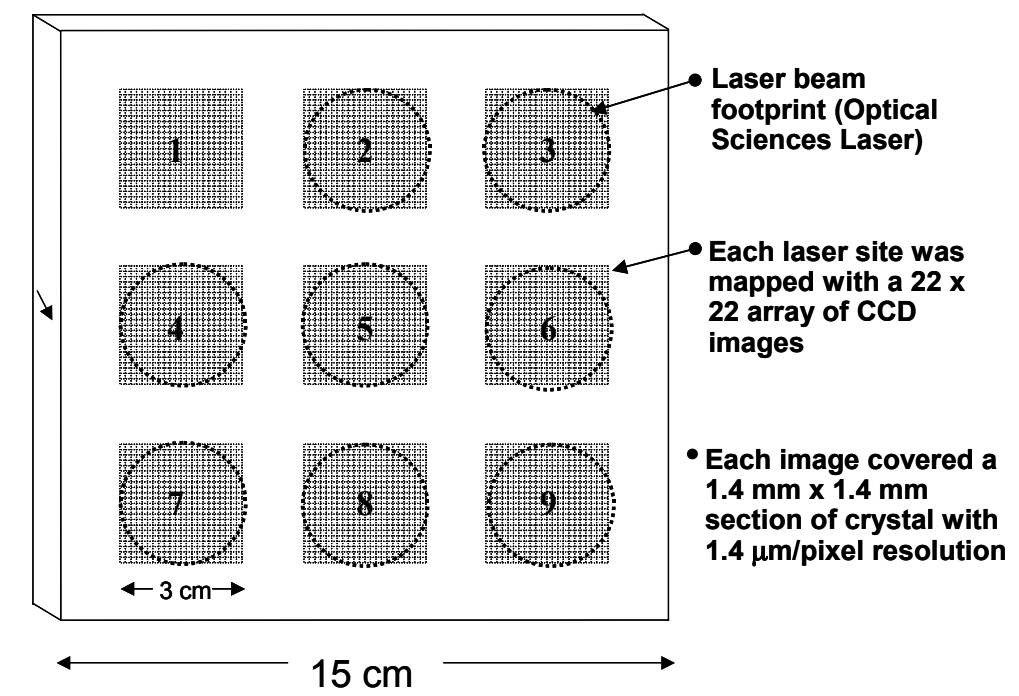

Figure 13: The crystal was mapped at each of the 9 sites shown above. The laser irradiated sites 2-9. Site 1 was left un-irradiated for reference.

Since bulk damage needed to be avoided for our surface damage experiments, it was first laser-conditioned. ${ }^{13,14} \mathrm{~A}$ five-shot ramp to $7 \mathrm{~J} / \mathrm{cm}^{2}$ was performed (nominally $1,3,5,6,7 \mathrm{~J} / \mathrm{cm}^{2}$ ) at each footprint. The actual fluence history for each footprint is shown in Table 2 .

Table 2: Average fluence for each shot of a five-shot ramp (351-nm, 3-ns) at each of the eight OSL beam footprints shown in Figure 13. The peak-to-valley intensity variation was about $\pm 15 \%$.

\begin{tabular}{|c|c|c|c|c|c|}
\cline { 2 - 6 } \multicolumn{1}{c|}{} & \multicolumn{5}{c|}{ Average Fluence $\left(\mathrm{J} / \mathrm{cm}^{2}\right)$} \\
\hline Test Site \# & Shot 1 & Shot 2 & Shot 3 & Shot 4 & Shot 5 \\
\hline $\mathbf{2}$ & 1.5 & 3.0 & 4.7 & 6.5 & 7.5 \\
\hline $\mathbf{3}$ & 1.4 & 3.3 & 5.3 & 6.3 & 7.3 \\
\hline $\mathbf{4}$ & 1.3 & 3.2 & 5.8 & 6.6 & 7.4 \\
\hline $\mathbf{5}$ & 1.5 & 3.6 & 5.5 & 6.2 & 7.5 \\
\hline $\mathbf{6}$ & 1.4 & 3.2 & 5.8 & 6.4 & 7.5 \\
\hline $\mathbf{7}$ & 1.5 & 3.1 & 5.6 & 6.2 & 7.6 \\
\hline $\mathbf{8}$ & 1.2 & 4.1 & 5.8 & 6.2 & 7.1 \\
\hline $\mathbf{9}$ & 1.7 & 3.4 & 5.6 & 5.8 & 7.1 \\
\hline
\end{tabular}


The fluorescence mapping was performed using a high-resolution imaging system, where a single image consists of a $1.4 \times 1.4 \mathrm{~mm}^{2}$ section of the sample surface. With the (liquid nitrogen-cooled) $1024 \times 1024 \mathrm{CCD}$ array, this gives $\sim 1.4 \mu \mathrm{m} /$ pixel resolution. To cover the $3-\mathrm{cm}$ diameter laser beam footprint, a $22 \times 22$ square array of these fluorescence images were taken at each OSL footprint (see Figure 13). The exposure time for each image was 35 seconds. The laser used as the excitation source was a Q-switched Nd:YLF (Photonics Industries model DS10-351), operating at $3 \mathrm{kHz}$ with an average power of about $750 \mathrm{~mW}$ and pulse width of $20 \mathrm{~ns}$. The angle of incidence is near grazing, and the illumination area on the sample was much larger than the $1.96 \mathrm{~mm}^{2}$ contained in each image. An identical array of images was also taken with a white-light illumination source (fiber-coupled ring light) instead of the 351-nm laser. These scatter images indicate whether a fluorescing object also scatters light. An exposure time of 0.5 seconds was used to collect these images.

Figure 14 shows a large-area scatter map of the crystal after exposure to 351-nm pulses from the OSL. This image was taken with a 45 mega-pixel scanning CCD camera. The sample was illuminated from the side with 6 " light bars. This light is transmitted through the bulk of the sample by total internal reflection, and is scattered by defects at the surface or in the bulk. The location of the defect relative to the surface of the crystal is lost in the image since the depth of focus of the imaging system is long compared to the crystal thickness.

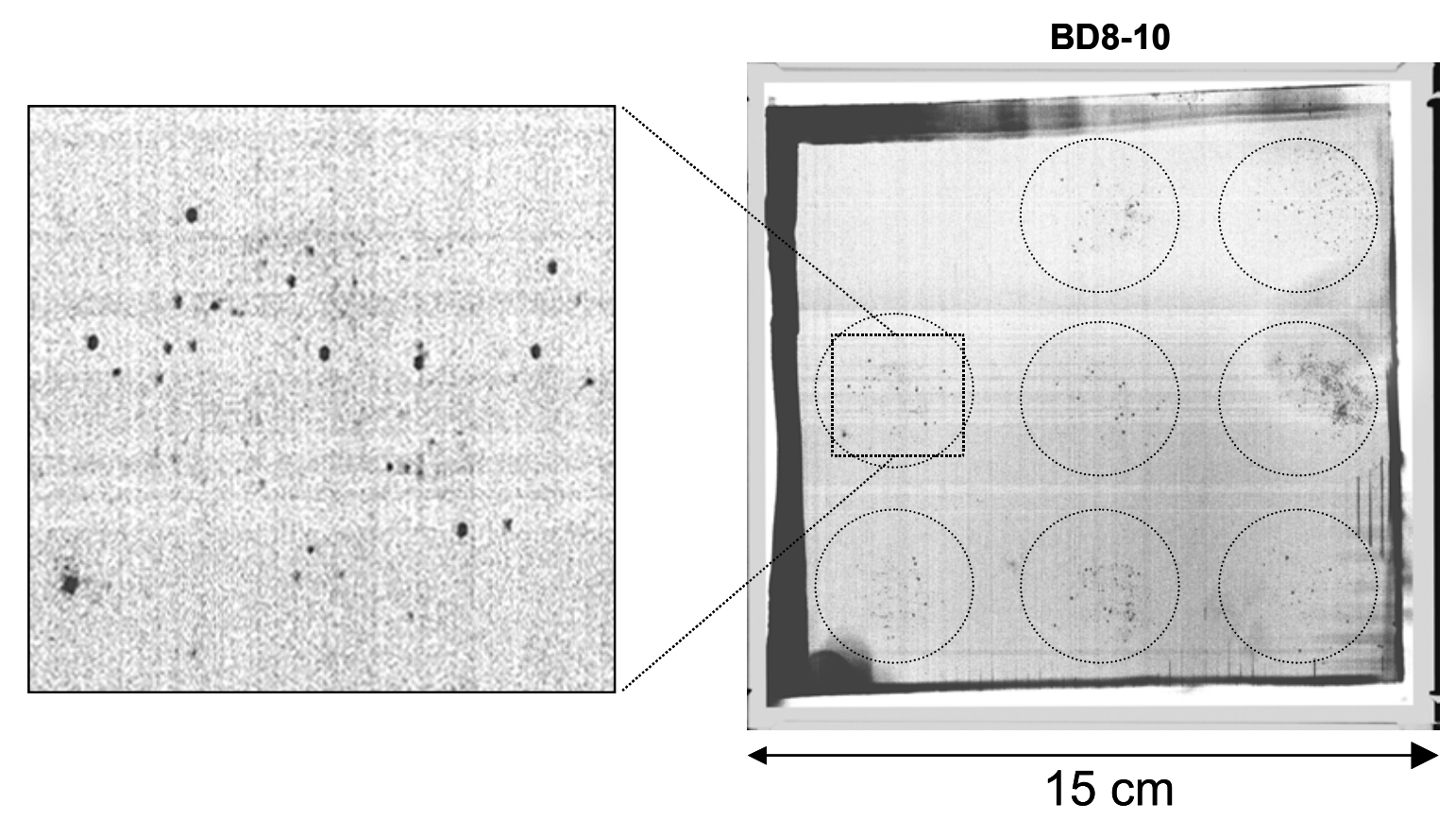

Figure 14: A large-area scatter map reveals output-surface damage (black areas) after exposure of sample BD8-10 to laser irradiation (351-nm, 3-ns) with successive shots up to $7 \mathrm{~J} / \mathrm{cm}^{2}$. Although input-surface, bulk, and outputsurface features are not distinguishable in these images, much of the damage shown at left is on the output surface. Site 6 shows the greatest amount of bulk damage. The vertical features between sites 6 and 9 are due to marks left over from the diamond turning process. These are removed with minimal laser fluence, for example by the first shot of the laser ramp. Most of the other features are artifacts of the imaging process.

The inset of Figure 14 shows a typical damage density in one 351-nm laser irradiation footprint. Most of the defects in this image are pits on the output surface. This data indicates an output-surface damage density on the order $2 / \mathrm{cm}^{2}$. Figure 15 shows brightfield microscope images of typical defect sites. These defect sites were typically pits $\sim 10$ microns in radius and $\sim 1$ micron in depth. 

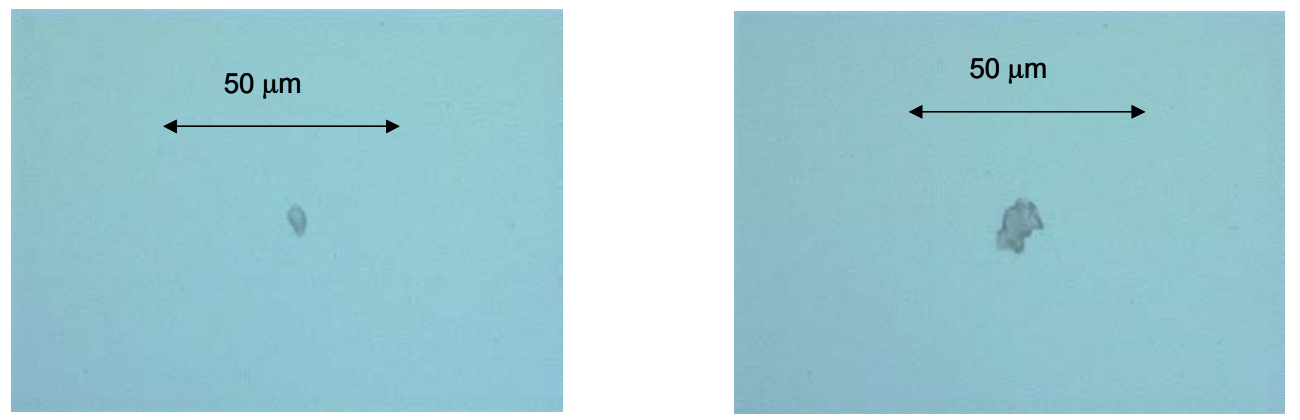

Figure 15: These microscope images show typical output surface pits after exposure to ramped fluences up to $7 \mathrm{~J} / \mathrm{cm}^{2}$ (351-nm, 3-ns) in sample BD8-10.

The correlation of the surface damage pits to precursors recorded in the fluorescence and scatter maps is displayed in the Venn diagram of Figure 16. Of the 54 pits identified, 38 had both a scatter and fluorescence precursor, 8 had only a fluorescence precursor, and another 8 had no precursor. These uncorrelated sites could be due to near-surface bulk damage, or by precursors that appeared after the maps were obtained but before the laser was fired, for example during the handling of the sample between the mapping and laser facilities.

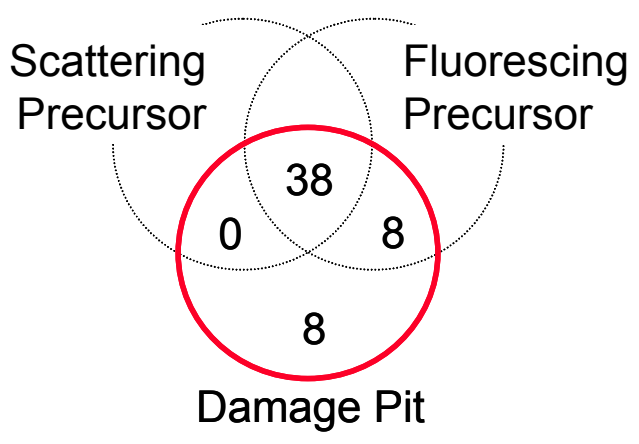

Figure 16: Venn diagram showing the correlation of output-surface damage pits to fluorescence and scatter precursors. A total of 54 damage pits were counted.

Of the 46 damage pits that were correlated to a precursor, 41 of these precursors $(90 \%)$ had a unique geometrical fluorescence signature. While most of these precursors also had a scatter signal as well, it was not nearly as distinctive as the fluorescence signal. Figure 17 shows the scatter and fluorescence images before irradiation up to 7 $\mathrm{J} / \mathrm{cm}^{2}$, and the scatter image after irradiation with the OSL laser. A magnified view of the precursor reveals its unique elliptical shape. They ranged from 10-20 microns in length. A nearby fluorescent feature, with a shape and size typical of those seen in general on these samples, did not lead to damage. 


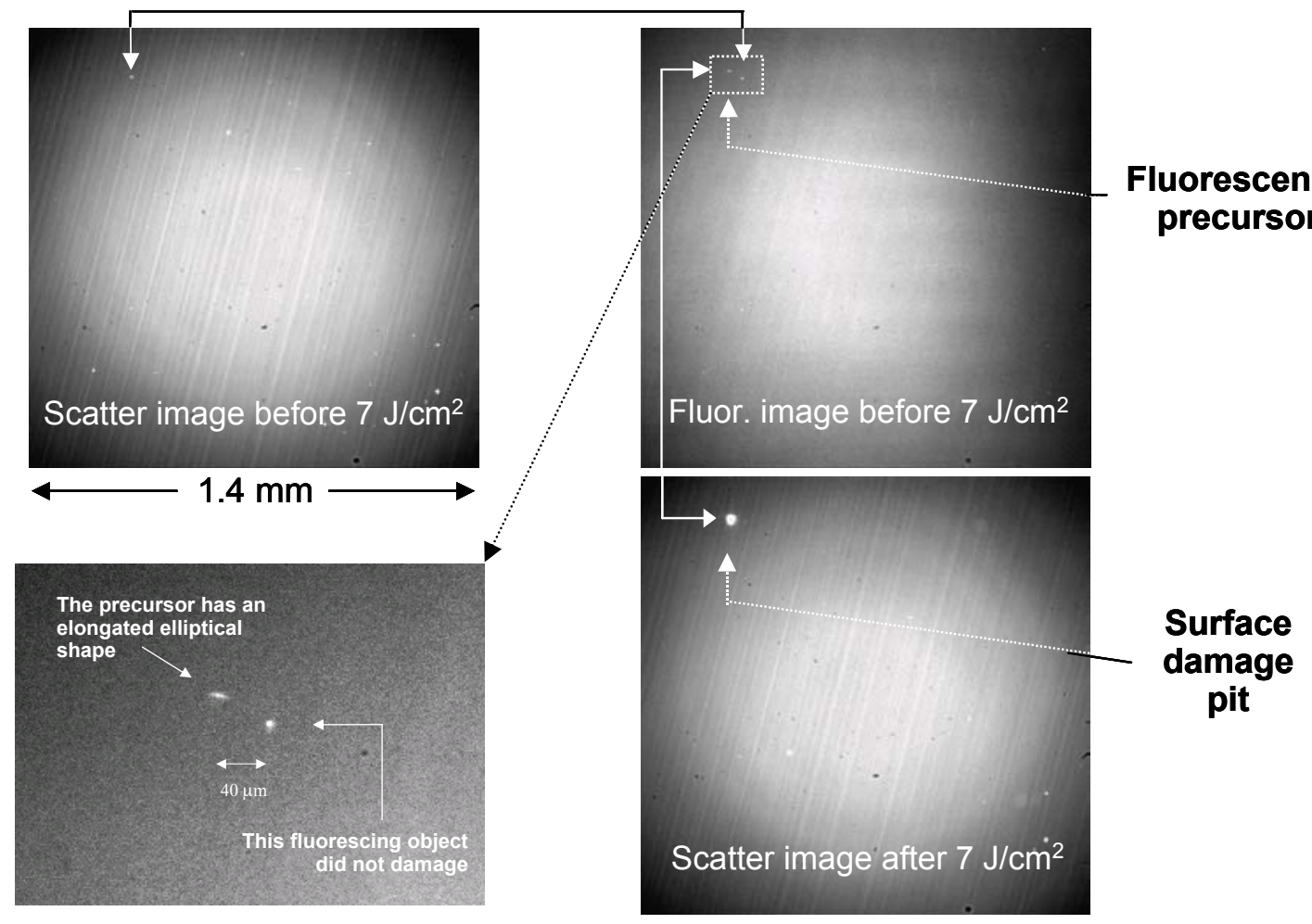

Figure 17: Before and after images showing a fluorescence precursor that led to damage after 351-nm exposure up to $7 \mathrm{~J} / \mathrm{cm}^{2}$. This fluorescence precursor had no scatter signal. A nearby feature both scattered and fluoresced. A magnified view of both features is shown in the lower left image.

In addition, most of these precursors were aligned perpendicular to the extraordinary axis of the crystal. We are currently investigating the nature and origin of these features. It is unclear whether they were incorporated during the growth of the crystal, or were created by mechanical means during finishing or handling. We are also investigating whether this phenomena is unique to this particular rapid-growth boule, or occurs more generally in all or most crystals.

\section{CONCLUSION}

The fluorescence images of $\mathrm{SiO}_{2}$ and DKDP samples demonstrate that this technique can detect the presence of contaminants embedded into the surface during the polishing process. There may be particular contaminants that are not emissive and therefore visible using fluorescence imaging. However, if the polishing process is such that contamination of the surface is a problem, fluorescence imaging may be a useful quality control tool.

Results from in-situ damage testing experiments suggest a correlation between visible fluorescing features and damage initiation. Furthermore, fluorescing objects located on the surface of the sample were shown to violently interact with the high fluence laser irradiation. In the high damage threshold sample, most of the fluorescing objects did not initiate damage at $\approx 15 \mathrm{~J} / \mathrm{cm}^{2}$ at 351 -nm, 3-ns. This indicates that this technique cannot be used as a uniquely identify damage precursors. The damage initiators may be visible with fluorescence imaging but not all observed features will initiate damage. Thus, damage initiation may be the result not only of absorption but also a set of other parameters such as electronic structure of the absorbent which can be critical for the initiation of subsequent multiphoton or multi-step electron excitation which can lead to cascade photo-ionization and plasma formation. The shape and thickness of the absorbing contaminant and its physical coupling with the host material are probably also important parameters.

The experimental results shown in Figures 11 and 12 demonstrate a conditioning effect where absorbing contaminants are removed from the surface without causing damage. This may be suggesting that sub-damage threshold illumination of the surface can remove contaminants that could initiate damage at a higher fluence. The mechanism for such effect may be that if the energy absorbed by some contaminants is barely sufficient to cause a micro-explosion associated with plasma formation and/or heating under 351-nm irradiation, the energy density 
(within the contaminant) may not be sufficient to cause significant energy transfer into the host that can lead to damage initiation.

The experiments on the $15-\mathrm{cm}$ DKDP crystal validated the usefulness of the fluorescence imaging technique for precursor detection. With this method, fluorescence features were identified and correlated to output surface damage pits after exposure up to $7 \mathrm{~J} / \mathrm{cm}^{2}$ at $351-\mathrm{nm}$ and $3-\mathrm{ns}$. $85 \%$ of all damage pits correlated to fluorescence precursors, and $90 \%$ of these exhibited an elliptically shaped unique fluorescence signal. The (15\%) uncorrelated pits could be due to near surface bulk damage or contaminants that appeared after the 'before' images were taken. Investigation is currently underway to chemically identify the unique fluorescence precursors, so that their origin can be determined. Removal of these features from the crystal (e.g. from the growth or finishing process) could lead to a significant decrease in the surface damage density of DKDP triplers.

\section{ACKNOWLEDGEMENTS}

This work was performed under the auspices of the U.S. Department of Energy by the University of California, Lawrence Livermore National Laboratory under contract No. W-7405-Eng-48.

\section{REFERENCES}

1. N. Bloembergen, "Role of cracks, pores, and absorbing inclusions on laser induced damage threshold at surfaces of transparent dielectrics", Applied Optics 12, 661-664 (1973).

2. J. Glass and A. H. Guenther, "Laser induced damage of optical elements-a status report", Applied Optics 12, 637-649 (1973).

3. J. A. Ringlien, N. L. Boling, and G. Dube, "An acid treatment for raising the surface damage threshold of laser glass", Applied Physics Letters 25, 598-600 (1974).

4. R. A. House, II, J. R. Bettis, and A. H. Guenther, "Efficacy of ion polishing optical surfaces", Applied Optics 16, 1486-1488 (1977).

5. P. A. Temple, W. H. Lowdermilk, and D. Milam, "Carbon dioxide laser polishing of fused silica surfaces for increased laser-damage resistance at 1064 nm”, Applied Optics 21, 3249-3255 (1982).

6. F.Y. Genin, M.D. Feit, M. R. Kozlowski, A.M. Rubenchik, A. Salleo, J. Yoshiyama, "Rear-surface laser damage on 355-nm silica optics owing to Fresnel diffraction on front-surface contamination particles" , Appl. Opt., 39, 3654, 2000

7. M. D. Feit, A. M. Rubenchik D. Faux , R. Riddle, A. Shapiro, D. C. Eder, B. M. Penetrante, D. Milam, F. Y. Genin, M. R. Kozlowski, Modeling of laser damage initiated by surface contamination, Optical materials for high power lasers, A. H. Guenther, Ed., SPIE, 3966, 417 (1996).

8. M. R. Kozlowski, J. Carr, I. D. Hutcheon, R. Torres, L. M. Sheehan, D. W. Camp, and M. Yan, "Depth profiling of polishing-induced contamination on fused silica surfaces", Laser-Induced Damage in Optical Materials, SPIE vol. 3244, 365-375, (1998).

9. G. J. Brakenhoff, H. T. M. van der Voort, E. A. van Spronsen, and N. Nanninga, "Three-dimensional imaging in fluorescence in confocal scanning microscopy," Journal of Microscopy, 153, 2, 151-159 (1989).

10. S. G. Demos, M. Staggs, M. Yan, H. B. Radousky and J. J. De Yoreo, "Microscopic fluorescence imaging of bulk defect clusters in $\mathrm{KH}_{2} \mathrm{PO}_{4}$ crystals", Opt. Lett., 24, 268 (1999).

11. S. G. Demos, M. R. Kozlowski, M. Staggs, L. L. Chase, A. Burnham, H. B. Radousky, "Mechanisms to explain damage growth in optical materials", Laser-Induced Damage in Optical Materials, G. J. Exarhos, A. H. Guenther, M. R. Kozlowski, K. L. Lewis, M. J. Soileau, Eds., Proc. SPIE, 4347, 277, (2000).

12. M. D. Feit, A. M. Rubenchik, M. Runkel, "Analysis of bulk DKDP damage distribution, obscuration and pulse length dependence," Laser-Induced Damage in Optical Materials, G. J. Exarhos; A. H. Guenther, M. R. Kozlowski; K. L. Lewis; M. J. Soileau; Eds., Proc. SPIE Vol. 4347, 383, (2000).

13. L.J. Atherton, F. Rainer, J.J. De Yoroe, I.M. Thomas, N. Zaitseva and F. De Marco, "Thermal and Laser conditioning of production and rapid-growth KDP and KD*P crystals," Laser-Induced Damage in Optical Materials, SPIE Proceedings, Vol. 2114, pp. 36-45, 1993.

14. M. Runkel, J.J. De Yoreo, W.D. Sell, D. Milam, "Laser conditioning study of KDP on the Optical Sciences Laser using large area beams," Laser-Induced Damage in Optical Materials, SPIE Proceedings, Vol. 3244, pp. 51-63, 1997. 


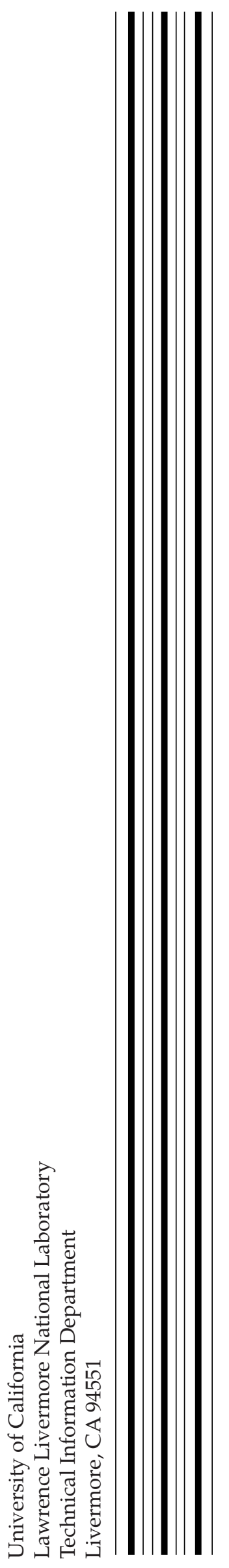

